\title{
Pattern of abdominal wall herniae in females: a retrospective analysis.
}

\author{
Olalekan Olasehinde, Amarachukwu C Etonyeaku., Elugwaraonu A Agbakwuru, \\ Ademola O Talabi, Funmilola O Wuraola, Adebayo G Tanimola.
}

Department of surgery,Obafemi Awolowo University Teaching Hospitals Complex, PMB 5538, Ile-Ife, Nigeria

\begin{abstract}
Background: Gender differences are expected to influence the pattern and outcome of management of abdominal wall hernias. Some of these are left to speculations with few published articles on hernias in females.

Objectives: To describe the clinical pattern of abdominal wall hernias in females.

Method: A 5 year retrospective review.

Result: There were 181 female patients with 184 hernias representing $27.9 \%$ of the total number of hernia patients operated. Mean age was $41.66 \pm 24.46$ years with a bimodal peak in the $1^{\text {st }}$ and $7^{\text {th }}$ decades. Inguinal hernia accounted for majority $(50.5 \%)$ but incisional hernia predominated in the 30-49 age group, while only inguinal and umbilical hernias were seen in the first two decades $(\mathrm{p}=0.04)$. There was no side predilection in the cases of inguinal hernia. There were $12(6.6 \%)$ emergency presentations, most of which occurred in the 6th decade and above and none below 30 years $(\mathrm{p}=0.02)$. Umbilical ( 4 cases) and femoral hernias (3cases) accounted for most of these cases. Incisional hernia was the commonest cause of recurrent hernias.

Conclusion: Inguinal hernia is the commonest hernia type in females followed by incisional hernias which also accounteds for most recurrent cases. Age appears to be a risk factor for developing complications.

Keywords: Female, hernia.

DOI: http://dx.doi.org/10.4314/ahs.v16i1.33

Cite as: Olasehinde O, Etonyeaku AC, Agbakwuru EA, Talabi AO, Wuraola FO, Tanimola AG. Pattern of abdominal wall herniae in females: a retrospective analysis. Afri Health Sci. 2016;16(1): 250- 254. http:// dx.doi.org/10.4314/abs.v16i1.33
\end{abstract}

\section{Introduction}

Hernias have been extensively studied over the years owing to their relative frequency worldwide ${ }^{1}$. Over the years, hernia management has witnessed improvements with the development of several techniques. Most available studies on hernias are markedly skewed towards the assessment the efficacy of these techniques with relatively fewer studies assessing other aspects of the subject of abdominal wall hernias ${ }^{2}$. One of such areas where there is relatively sparse information is the pattern of abdominal wall hernias relating specifically to gender. It is not unexpected to find variations in the pattern of hernia presentation and outcome of management between males and females owing to the

\footnotetext{
Corresponding author:

Olalekan Olasehinde,

Department of surgery,

Obafemi Awolowo University

Teaching Hospitals Complex,

PMB 5538, Ile-Ife, Nigeria.

Mobile number: +2348060716670

Email: lekanolasehinde@yahoo.com
}

anatomical, embryological and physiological differences that exist between them.

Some of these have been left to speculations and assumptions with our search revealing very few published literature in this regard. This study set out to review the characteristics of female hernia patients with the aim of describing the demographics, pattern of abdominal wall herniation, mode of presentation, treatment and recurrence following repair.

\section{Methodology}

A descriptive retrospective review of all female patients who had hernia repair at Obafemi Awolowo University Teaching Hospitals Complex Ile-Ife, Nigeria which is a 600 bed capacity hospital in South-Western Nigeria that offers tertiary care. The study was carried out between January 2009 and December 2013.

Record of all female patients who had hernia repair during the study was obtained from the medical records. Details of their socio-demographic characteristics, hernia type, mode of presentation, method of repair and outcome particularly recurrence were obtained. The duration of follow up documented in the 
case records ranged from 1 week to 6 years. Follow up was by clinical evaluation at the out-patient department. Data analysis was done using SPSS version 22, Chicago inc. Data was expressed as means, simple frequencies, graphs and charts.

\section{Results}

A total of 649 hernia patients were seen during the study period. There were 181 females with 184 hernias representing $27.9 \%$ of the total. Their ages ranged from 1 week to 90 years with a mean age of $41.66 \pm 24.46$ years. The age distribution of the patients as depicted in figure 1 shows a bimodal peak.

Figure 1: Age distribution (showing a bimodal peak)

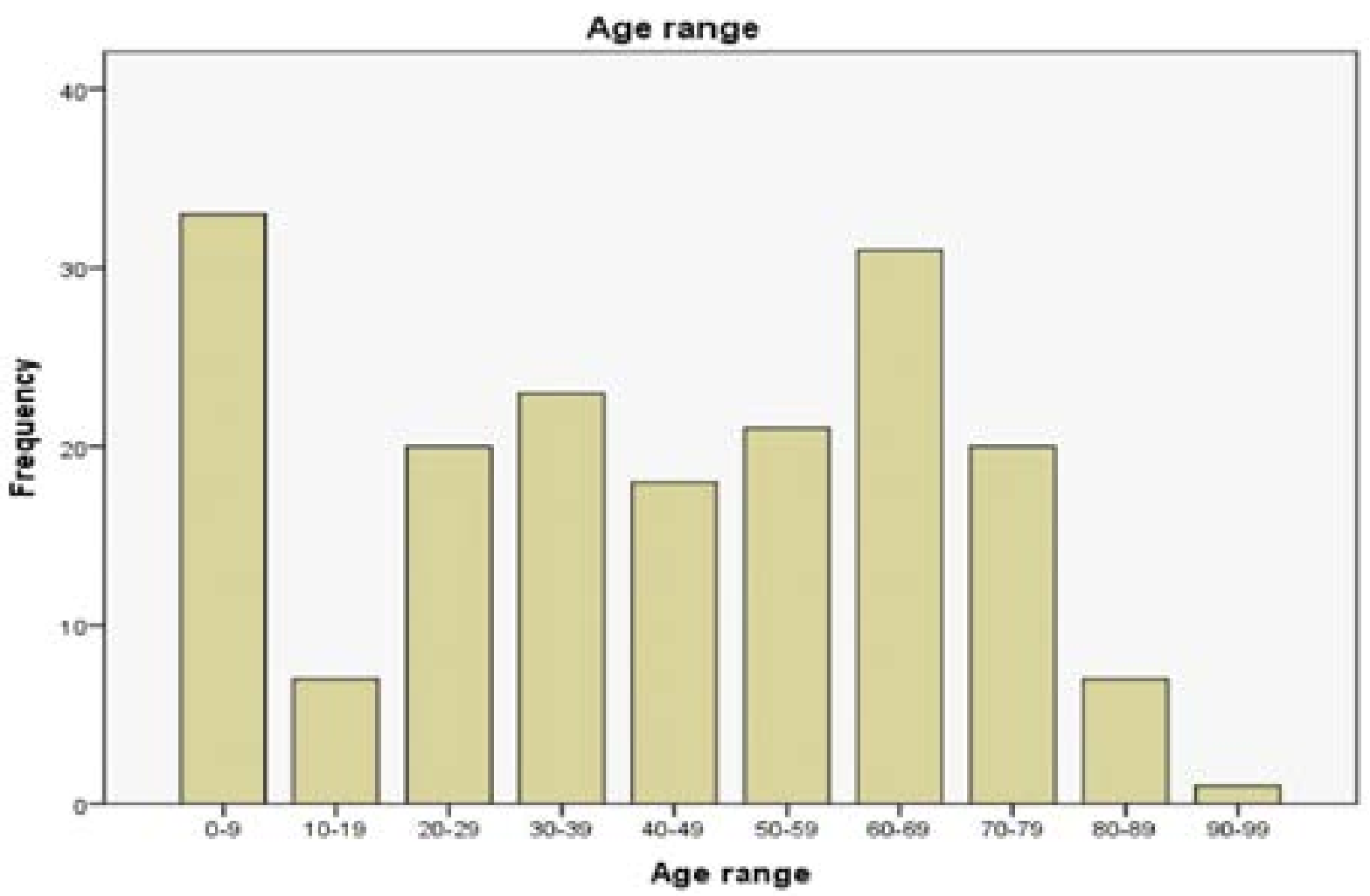

While hernias were found across all age groups, there was a significant difference in the type of hernias found, $(p=0.04)$ with inguinal and umbilical hernias being the only types noted among patients 20 years and below. Overall, inguinal hernias accounted for majority of cases followed by incisional hernias (Table 1).

\begin{tabular}{|lcc|}
\hline \multicolumn{3}{|c|}{ Table 1: Distribution of Abdominal wall hernias } \\
\hline \hline Hernia type & Frequency & \\
& Percentage & \\
\hline Inguinal & 93 & 50.5 \\
Incisional & 27 & 14.7 \\
Umbilical & 21 & 11.4 \\
Epigastric & 19 & 10.3 \\
Paraumbilical & 11 & 6 \\
Femoral & 11 & 6 \\
Lumbar & 1 & 0.5 \\
Obturator & 1 & 0.5 \\
\hline Total & $\mathbf{1 8 4}$ & $\mathbf{1 0 0}$ \\
\hline
\end{tabular}


When the various age groups were considered however, inguinal hernia still predominated in all age groups except among patients between 30-39 years who had incisional hernias predominating. Hernias were solitary in 172 patients $(95 \%)$ while 9 patients $(5 \%)$ had multiple hernias. All the patients with multiple hernias had inguinal hernia as one of the components. Majority $(169=93.4 \%)$ presented with reducible hernias while 12 patients $(6.6 \%)$ had emergency presentations. None of the emergency presentations occurred in patients less than 30 years while nine of the twelve emergency cases $(75 \%)$ occurred in patients in their 6th decade and above $(p=0.02)$. Inguinal hernia though the commonest overall, had only one emergency presentation, with umbilical (4 cases), femoral (3) incisional (2), epigastric (1) and obturator hernias (1) accounting for the others. In terms of proportion however, femoral hernias had the highest rate of obstruction with three of the eleven cases $(27.3 \%)$ presenting as emergencies. Most of the hernias were primary $(172=95 \%)$ while there were nine recurrent cases $(5 \%)$ with incisional hernias accounting for the highest number ( 4 patients $=44.4 \%$ ).

Of the 93 patients who had inguinal hernia, 9 were bilateral, 42 were left sided while 42 were right sided showing no difference with regard to laterality. The mode of treatment of the various hernias reveals that incisional hernia was the most common indication for the use of mesh with 22 of the 26 cases of incisional hernia $(84.6 \%)$ repaired with mesh. Only 19 of the 66 cases of adult inguinal hernias $(28.8 \%)$ were repaired with mesh prosthesis. Hernias were safely repaired in all the patients with no mortality recorded. The duration of follow up ranged from one week to six years with only one patient followed up for 6 years. There was a case of recurrence in a 75 year old patient following suture repair of an obstructed incisional hernia.

\section{Discussion}

The structural differences between males and females may cause variations in both frequency and pattern of hernias. This includes differences in the bony pelvis, the musculofascial layers of the lower abdomen and the descent of the gonads from the retroperitoneum ${ }^{3}$.

The predominance of inguinal hernia in this study certainly conforms to general knowledge $\mathrm{e}^{4}$ except that the usual right sided pre-ponderance observed in males which has also been reported by some authors among females to be in the range of $1.5: 15,{ }^{6}$ was not so observed in this study with equal number of right and left sided inguinal hernias. The basis for the right sided predominance in males which is the later descent of the testis on the right side is a phenomenon that is non-existent in females. Those who have observed right sided predominance have not been able to attribute it to any scientific hypothesis ${ }^{3}$ and as such it may well be considered a chance finding. The embryological basis for the development of inguinal and umbilical hernias explains why these were the only hernia types found in patients less than 20 years.

Generally, inguinal hernias have been known to be the commonest hernia type irrespective of gender with umbilical, epigastric and femoral hernias ranking next ${ }^{7}$. It is however noteworthy that incisional hernia ranked next to inguinal hernia in terms of frequency in this study and in fact more frequent than inguinal hernias in the 30-39 age group. This perhaps underscores the difference in the pattern of hernias observed in females compared with what is generally observed ${ }^{7}$. It may also be a reflection of the increase in the number of surgical operations, particularly obstetric and gynecological surgeries which are common in this group of patients. Most studies that have evaluated incisional hernias have also reported a higher incidence in women with majority occurring following obstetric surgeries ${ }^{8,9}$. Being a preventable type of hernia, the possibility of its occurrence as a complication of surgery must be taken into consideration and efforts made to prevent its occurrence as much as possible as the number and magnitude of surgical operations continue to increase.

Regarding emergency presentations, majority of the patients were elderly which agrees with what is generally known concerning the effect of age on the development of hernia complications ${ }^{10,11}$. Femoral and umbilical hernias accounted for majority of the complicated cases with femoral hernias having the highest proportion of its cases presenting as emergencies. This is however not surprising as femoral hernias have been known to strangulate more frequently than other hernias. Gellegos and colleagues noted that the cumulative probability of femoral hernia strangulation at 3 months and 21 months following diagnosis was $22 \%$ and $45 \%$ respectively compared to $3 \%$ and $4.5 \%$ respectively in inguinal hernias ${ }^{12}$. The relatively higher rate of complicated femoral hernias may in part be due to the low index of suspicion as some studies have shown that patients particularly females with femoral hernias are less likely to have a groin examination with as high as $40 \%$ of cases being missed ${ }^{13,14}$. 
The only case of obturator hernia in this study is noteworthy; it occurred in a 90 year old asthenic woman who had acute intestinal obstruction with no obvious identifiable cause. This fits into the typical description of what has been previously documented in literature, with majority typically found in elderly females with a female to male ratio of 6.1 to $1^{15,16}$. The horizontal nature of the obturator canal in females perhaps accounts for their predisposition ${ }^{3}$. Since the hernia is usually not palpable, the diagnosis is usually made intra-operatively. As such a high index of suspicion is required to make a diagnosis and it should be considered when an elderly asthenic female patient presents with intestinal obstruction with no readily identifiable cause.

A significant number of the recurrent cases in the study were accounted for by incisional hernias, all of whom had failed non-prosthetic repairs. Various studies have also observed higher recurrence rates following non-prosthetic incisional hernia repair compared with mesh repair thus underscoring the growing preference for mesh repair techniques ${ }^{17,18}$. This fact is also reflected in this study with incisional hernia accounting for most cases of mesh prosthesis usage in females. Inguinal hernias, though commonest were mostly repaired using suture repair techniques with no recurrence noted. Thairu et al in their study on open inguinal hernias in females questioned the routine use of mesh for open repair of inguinal hernias in females and concluded that its routine use for open indirect hernia repair in females may be unnecessary ${ }^{19}$. There was only one case of recurrence noted in this study which occurred in an elderly patient who presented with obstructed incisional hernia which precluded the use of polypropylene mesh prosthesis because of the risk of mesh infection. She thus had direct suture repair which is expectedly associated with a high recurrence rate. What is currently advocated in such instances is the use of absorbable mesh ${ }^{20}$.

\section{Limitation}

The retrospective nature of this study and the lack of a standardized follow up schedule may not have allowed for adequate surveillance following surgery and as such the documented recurrence rate is within the limits of the data available in the case records.

\section{Conclusion}

Inguinal hernias are the commonest hernia type in females in this study except in the 4th decade of life where incisional hernias predominate. Elderly females particularly those with femoral hernias had the highest risk of emergency presentation. Incisional hernia accounts for most cases of recurrent hernias and it is the commonest indication for mesh usage in female hernia operations.

\section{References}

1. Kingsworth A, Le Blanc K. Hernias: inguinal and incisional. Lancet 2003; 362:1561-71

2. Lars N J, Finn G. The role of collagen in hernia genesis. In. Bendavid $\mathrm{R}$ et al(ed). Abdominal wall hernias, principles and management. 1st ed. New york: Springer verlag: 2001. 150-155

3. Alejandro W, SalvadorV, Denzil G, Alfredo B. Epidemiology of hernias in female. In Bendavid R. et al, (ed). Abdominal wall hernias, principles and management.1st Ed. New York: Springer verlag: 2001.613-619

4. Dabbas N, Adams K, Pearson K, Royle G. Frequency of abdominal wall hernias: is classical teaching out of date? JRSM Short Rep. 2011. 19;2(1):5.

5. Nordback I. Side incidence of inguinal hernias. Ann Chir Gynaecol. 1984; 73: 87-90.

6. Czeizel A. Epidemiologic characteristics of congenital inguinal hernia. Helv Paediatr Acta. 1980;35:57-67.

7. Natalie Dabbas K Adams K Pearson GT Royle. Frequency of abdominal wall hernias: Is classical teaching out of date? JRSM short reports. 2011; 2(1):5.

8. EA Agbakwuru, JK Olabanji, OI Alatise, RO Okwerekwu, OA Esimai. Incisional Hernia In Women: Predisposing Factors and Management Where Mesh is Not Readily Available. Libyan Journal of Medicine. 2008;4(2):66-69

9. Rutkow IM1, Robbins AW. Demographic, classificatory, and socioeconomic aspects of hernia repair in the United States. Surg Clin North Am. 1993;73(3):413-26

10. Broll R, Weisser C, Mühlschlegel M. Inguinal hernia in old age. Dtsch Med Wochenschr. 1987 ;112(16):641-3.

11. Mark D, Davies C, Morris-Stiff G, Shute K. Emergency Presentation of Abdominal Engl. Hernias: Outcome and Reasons for Delay in Treatment - A Prospective Study. Ann R Coll Surg 2007; 89(1): 47-50.

12. Gellegos NC, Dawson J, Jarvis M, Hobsley M. Risk of strangulation in groin hernias. $\mathrm{Br} J$ Surg. 1991;78:1171-3.

13. Nilsson H, Nilsson E, Angeras U, Nordin P. Mortality after groin hernia surgery: Delay of treatment and cause of death. Hernia 2011; 15:301-7

14. Kjaergaard J, Nielson M, Kehlet M, Mortality following emergency groin hernia surgery in Denmark. Hernia 2010; 14: 351-5.

15. Skandalakis JE, Gray SW, Burns WB, et al. Internal 
and external supravesical hernia. Am J Surg. 1976;42:142. MH.Suture versus mesh repair for incisional hernia. 16. Solli B, Bobbio A, Di Gioia MV, et al. The obturator hernia: a diagnostic challenge? Acta Biomed Arteneo Parmense. 1994;65:235-240.

17. Luijendijk RW, Hop WC, van den Tol MP, de Lange DC, Braaksma MM, IJzermans JN et al. A comparison of suture repair with mesh repair for incisional hernia. New Engl J Med. 2000; 343(6):392-8 Saudi Med J. 2006;27(5):652-6.

19. Thairu NM, Heather BP, Earnshaw JJ. Open inguinal hernia repair in women: is mesh necessary? Hernia. $2008 ; 12(2): 173-5$

20. G Campanelli, F Catena, and L Ansaloni Prosthetic abdominal wall hernia repair in emergency surgery: from polypropylene to biological meshes. World J Emerg 18. Al-Salamah SM, Hussain MI, Khalid K, Al-Akeely Surg. 2008; 3: 33. 